\title{
On subgroups in division rings of type 2
}

\author{
Bui Xuan Hai, Trinh Thanh Deo, and Mai Hoang Bien ${ }^{\ddagger}$
}

\begin{abstract}
Let $D$ be a division ring with center $F$. We say that $D$ is a division ring of type 2 if for every two elements $x, y \in D$, the division subring $F(x, y)$ is a finite dimensional vector space over $F$. In this paper we investigate multiplicative subgroups in such a ring.
\end{abstract}

Key words: division ring; type 2; finitely generated subgroups.

Mathematics Subject Classification 2010: 16K20

\footnotetext{
${ }^{*}$ Corresponding author, Faculty of Mathematics and Computer Science, University of Science, VNUHCM, 227 Nguyen Van Cu Str., Dist. 5, HCM-City, Vietnam, e-mail: bxhai@hcmus.edu.vn

${ }^{\dagger}$ Faculty of Mathematics and Computer Science, University of Science, VNU-HCM, 227 Nguyen Van Cu Str., Dist. 5, HCM-City, Vietnam, e-mail: ttdeo@hcmus.edu.vn

${ }^{\ddagger}$ Department of Basic Sciences, University of Architecture, 196 Pasteur Str., Dist. 1, HCM-City, Vietnam, e-mail: maihoangbien012@yahoo.com
} 


\section{Introduction}

In the theory of division rings, one of the problems is to determine which groups can occur as multiplicative groups of non-commutative division rings. There are some interesting results relating to this problem. Among them we note the famous discovery of Wedderburn in 1905, which states that if $D^{*}$ is a finite group, then $D$ is commutative, where $D^{*}$ denotes the multiplicative group of $D$. Later, L. K. Hua (see, for example, in [12, p. 223]) proved that the multiplicative group of a non-commutative division ring cannot be solvable. Recently, in [8] it was shown that the group $D^{*}$ cannot even be locally nilpotent. Note also Kaplansky's Theorem (see $\left[12,(15.15)\right.$, p. 259]) which states that if the group $D^{*} / F^{*}$ is torsion, then $D$ is commutative, where $F$ is the center of $D$. Some other results of this kind can be found for example, in [1]-[3], [6]-[8],...

In this paper we consider this question for division rings of type 2. Recall that a division ring $D$ with center $F$ is said to be division ring of type 2 if for every two elements $x, y \in D$, the division subring $F(x, y)$ is a finite dimensional vector space over $F$. This concept is an extension of that of locally finite division rings. By definition, a division ring $D$ is centrally finite if it is a finite dimensional vector space over its center $F$ and $D$ is locally finite if for every finite subset $S$ of $D$, the division subring $F(S)$ generated by $S \cup F$ in $D$ is a finite dimensional vector space over $F$. There exist locally finite division rings which are not centrally finite (it is not hard to give some examples). Of course, every locally finite division ring is a ring of type 2 . However, at present no example of a division ring of type 2 is known which is not locally finite. The difficulties are related with the following famous longstanding conjecture known as the Kurosh Problem for division rings [11. Recall that a division ring $D$ is algebraic over its center $F$ (briefly, $D$ is algebraic), if every element of $D$ is algebraic over $F$. Clearly, a locally finite division ring is algebraic. Kurosh conjectured that any algebraic division ring is locally finite. Unfortunately, this problem remains still unsolved in general, it is answered in the affirmative for the following special cases: for $F$ uncountable [14], $F$ finite [12], and for $F$ having only finite algebraic field extensions (in particular for $F$ algebraically closed). The last case follows from the Levitzki-Shirshov Theorem which states that any algebraic algebra of bounded degree is locally finite (see e.g. [5], [11]). The answer for the case of finite $F$ is due to Jacobson who proved that an algebraic division ring $D$ is commutative provided its center is finite (see, for example, [12]). Later, more general theorems of this kind (known as commutativity theorems) were proved by Jacobson and Herstein. For more information we refer to [9, Ch. 3]. Finally, we would like to note that the results obtained in this paper for division rings of type 2 have not been proved elsewhere before for locally finite division rings. So, at least (in the fortunate case if the Kurosh Problem will be answered in the affirmative, 
as we would like to see) our results generalize previous results for the finite dimensional case.

Throughout this paper the following notation will be used consistently: $D$ denotes a division ring with center $F$ and $D^{*}$ is the multiplicative group of $D$. If $S$ is a nonempty subset of $D$, then we denote by $F[S]$ and $F(S)$ the subring and the division subring of $D$ generated by $S$ over $F$, respectively. The symbol $D^{\prime}$ is used to denote the derived group $\left[D^{*}, D^{*}\right]$. An element $x$ in $D$ is said to be radical over a subring $K$ of $D$ if there exists some positive integer $n(x)$ depending on $x$ such that $x^{n(x)} \in K$. A nonempty subset $S$ of $D$ is radical over $K$ if every element of $S$ is radical over $K$. We denote by $N_{D / F}$ and $R N_{D / F}$ the norm and the reduced norm, respectively. Finally, if $G$ is any group then we always use the symbol $Z(G)$ to denote the center of $G$.

\section{Finitely generated subgroups}

The main purpose in this section is to prove that in any non-commutative division ring of type 2 there are no finitely generated subgroups containing the center.

Lemma 2.1. Let $D$ be a division ring with center $F, D_{1}$ be a division subring of $D$ containing $F$. Suppose that $D_{1}$ is a finite dimensional vector space over $F$ and $a \in D_{1}$. Then, $N_{D_{1} / F}(a)$ is periodic if and only if $N_{F(a) / F}(a)$ is periodic.

Proof. Let $F_{1}=Z\left(D_{1}\right) \supset F, m^{2}=\left[D_{1}: F_{1}\right]$ and $n=\left[F_{1}(a): F_{1}\right]$. By [4, Lemma 3, p.145] and [4, Corollary 4, p. 150], we have

$$
N_{D_{1} / F_{1}}(a)=\left[R N_{D_{1} / F_{1}}(a)\right]^{m}=\left[N_{F_{1}(a) / F_{1}}(a)\right]^{m^{2} / n} .
$$

Now, using the Tower formulae for the norm (cf. [4]), from the equality above we get

$$
N_{D_{1} / F}(a)=\left[N_{F_{1}(a) / F}(a)\right]^{m^{2} / n}
$$

Since $a \in F(a)$, wehave $N_{F_{1}(a) / F(a)}(a)=a^{k}$, where $k=\left[F_{1}(a): F(a)\right]$. Therefore

$$
N_{F(a) / F}\left(a^{k}\right)=N_{F(a) / F}\left(N_{F_{1}(a) / F(a)}(a)\right)=N_{F_{1}(a) / F}(a) .
$$

It follows that $N_{D_{1} / F}(a)=\left[N_{F(a) / F}(a)\right]^{k m^{2} / n}$, and the conclusion is now obvious.

The following proposition is useful. In particular, it is needed to prove the subsequent theorem.

Proposition 2.2. Let $D$ be a division ring with center $F$. If $N$ is a subnormal subgroup of $D^{*}$ then $Z(N)=N \cap F$. 
Proof. If $N$ is contained in $F$ then there is nothing to prove. Thus, suppose that $N$ is non-central. By $\left[15,14.4 .2\right.$, p. 439], $C_{D}(N)=F$. Hence $Z(N) \subseteq N \cap F$. Since the inclusion $N \cap F \subseteq Z(N)$ is obvious, $Z(N)=N \cap F$.

Theorem 2.3. Let $D$ be a division ring of type 2. Then $Z\left(D^{\prime}\right)$ is a torsion group.

Proof. By Proposition 2.2, $Z\left(D^{\prime}\right)=D^{\prime} \cap F^{*}$. Any element $a \in Z\left(D^{\prime}\right)$ can be written in the form $a=c_{1} c_{2} \ldots c_{r}$, where $c_{i}=\left[x_{i}, y_{i}\right]$ with $x_{i}, y_{i} \in D^{*}$ for $i \in\{1, \ldots, r\}$. Put $D_{1}=D_{2}:=F\left(c_{1}, c_{2}\right), D_{3}:=F\left(c_{1} c_{2}, c_{3}\right), \ldots, D_{r}:=F\left(c_{1} \ldots c_{r-1}, c_{r}\right)$ and $F_{i}=Z\left(D_{i}\right)$ for $i \in\{1, \ldots, r\}$. Since $D$ is of type $2,\left[D_{i}: F\right]<\infty$.

Since $N_{F\left(x_{i}, y_{i}\right) / F}\left(c_{i}\right)=1$, by Lemma 2.1, $N_{F\left(c_{i}\right) / F}\left(c_{i}\right)$ is periodic. Again by Lemma 2.1, $N_{D_{i} / F}\left(c_{i}\right)$ is periodic. Therefore, there exists some positive integer $n_{i}$ such that $N_{D_{i} / F}\left(c_{i}^{n_{i}}\right)=$ 1. Recall that $D_{2}=D_{1}$. Hence we get

$$
N_{D_{2} / F}\left(c_{1} c_{2}\right)^{m}=N_{D_{2} / F}\left(c_{1}\right)^{m} N_{D_{2} / F}\left(c_{2}\right)^{m}=1,
$$

where $m=n_{1} n_{2}$. Again by Lemma 2.1, $N_{F\left(c_{1} c_{2}\right) / F}\left(c_{1} c_{2}\right)$ is periodic; hence $N_{D_{3} / F}\left(c_{1} c_{2}\right)$ is periodic. By induction, $N_{D_{r} / F}\left(c_{1} \ldots c_{r-1}\right)$ is periodic. Suppose that $N_{D_{r} / F}\left(c_{1} \ldots c_{r-1}\right)^{n}=1$. Then

$$
N_{D_{r} / F}\left(a^{n}\right)=N_{D_{r} / F}\left(c_{1} \ldots c_{r-1}\right)^{n} N_{D_{r} / F}\left(c_{r}\right)^{n}=1 .
$$

Hence, $a^{n\left[D_{r}: F\right]}=1$. Therefore, $a$ is periodic. Thus $Z\left(D^{\prime}\right)$ is torsion.

Corollary 2.4. Let $D$ be a non-commutative ring of type 2 with center $F$. Then $D^{\prime} \backslash Z\left(D^{\prime}\right)$ contains no elements purely inseparable over $F$.

Proof. Suppose that $a \in D^{\prime} \backslash Z\left(D^{\prime}\right)$ is purely inseparable over $F$. Then, there exists some positive integer $m$ such that $a^{p^{m}} \in F$. Since $Z\left(D^{\prime}\right)=D^{\prime} \cap F$ (by Proposition 2.2), $a^{p^{m}} \in Z\left(D^{\prime}\right)$. By Theorem 2.3, there exists some positive integer $r$ such that $a^{r p^{m}}=1$. Denote by $k$ the order of $a$ in the group $D^{*}$. If $p$ divides $k$, then $k=p t$ and we have

$$
1=a^{k}=a^{p t}=\left(a^{t}\right)^{p} .
$$

Consequently, $a^{t}=1$, which is impossible in view of the choice of $k$. Now, suppose that $p$ does not divide $k$. Then, $\left(k, p^{m}\right)=1$ and $\alpha k+\beta p^{m}=1$ for some integers $\alpha$ and $\beta$. Therefore, we have

$$
a=a^{\alpha k+\beta p^{m}}=\left(a^{k}\right)^{\alpha} \cdot a^{\beta p^{m}}=\left(a^{p^{m}}\right)^{\beta} \in F .
$$

Consequently, $a \in F \cap D^{\prime}=Z\left(D^{\prime}\right)$, a contradiction. 
Note that in 13 the author proved that $Z\left(D^{\prime}\right)$ is finite if $D$ is centrally finite. In virtue of this fact, he expressed his ideas that $Z\left(D^{\prime}\right)$ is torsion for any division ring $D$ algebraic over its center, but he has not been able to prove this. Therefore, Theorem 2.3 represents some progress in this direction. Moreover (and this is more important for our purpose), we need this theorem to establish the main result in the present section. In fact, we shall prove that in a division ring $D$ of type 2 with center $F$, there are no finitely generated subgroups containing $F^{*}$. Consequently, if $D$ is of type 2 and $D^{*}$ is finitely generated, then $D$ is a field. Note that if the multiplicative group of a field is finitely generated, then it is finite. So, if $D$ is of type 2 and $D^{*}$ is finitely generated, then $D$ is even a finite field. Our next theorem strongly generalizes the result obtained in [2, Theorem 1] which states that, if $D$ is centrally finite and $D^{*}$ is finitely generated, then $D$ is commutative.

Theorem 2.5. Let $D$ be a non-commutative division ring of type 2 with center $F$ and suppose that $N$ is a subgroup of $D^{*}$ containing $F^{*}$. Then $N$ is not finitely generated.

Proof. Suppose that there is a finitely generated subgroup $N=\left\langle x_{1}, x_{2}, \ldots, x_{n}\right\rangle$ of $D^{*}$ containing $F^{*}$. Then, in virtue of $\left[15,5.5 .8\right.$, p. 113], $F^{*} N^{\prime} / N^{\prime}$ is a finitely generated abelian group, where $N^{\prime}$ denotes the derived subgroup of $N$.

Case 1: $\operatorname{char}(D)=0$.

Then, $F$ contains the field $\mathbb{Q}$ of rational numbers and it follows that $\mathbb{Q}^{*} /\left(\mathbb{Q}^{*} \cap N^{\prime}\right) \simeq$ $\mathbb{Q}^{*} N^{\prime} / N^{\prime}$. Since $F^{*} N^{\prime} / N^{\prime}$ is finitely generated, $\mathbb{Q}^{*} N^{\prime} / N^{\prime}$ is finitely generated and consequently $\mathbb{Q}^{*} /\left(\mathbb{Q}^{*} \cap N^{\prime}\right)$ is finitely generated. Consider an arbitrary element $a \in \mathbb{Q}^{*} \cap N^{\prime}$. Then $a \in F^{*} \cap D^{\prime}=Z\left(D^{\prime}\right)$. By Theorem $[2.3$, $a$ is periodic. Since $a \in \mathbb{Q}$, we get $a= \pm 1$. Thus, $\mathbb{Q}^{*} \cap N^{\prime}$ is finite. Since $\mathbb{Q}^{*} /\left(\mathbb{Q}^{*} \cap N^{\prime}\right)$ is finitely generated, $\mathbb{Q}^{*}$ is finitely generated, which is impossible.

Case 2: $\operatorname{char}(D)=p>0$.

Denoting by $\mathbb{F}_{p}$ the prime subfield of $F$, we shall prove that $F$ is algebraic over $\mathbb{F}_{p}$. In fact, suppose that $u \in F$ and $u$ is transcendental over $\mathbb{F}_{p}$. Then, the group $\mathbb{F}_{p}(u)^{*} /\left(\mathbb{F}_{p}(u)^{*} \cap N^{\prime}\right)$ considered as a subgroup of $F^{*} N^{\prime} / N^{\prime}$ is finitely generated. Consider an arbitrary element $f(u) / g(u) \in \mathbb{F}_{p}(u)^{*} \cap N^{\prime}$, where $f(X), g(X) \in \mathbb{F}_{p}[X],((f(X), g(X))=$ 1 and $g(u) \neq 0$. As above, we have $f(u)^{s} / g(u)^{s}=1$ for some positive integer $s$. Since $u$ is transcendental over $\mathbb{F}_{p}$, it follows that $f(u) / g(u) \in \mathbb{F}_{p}$. Therefore, $\mathbb{F}_{p}(u)^{*} \cap N^{\prime}$ is finite and consequently, $\mathbb{F}_{p}(u)^{*}$ is finitely generated, so $\mathbb{F}_{p}(u)$ is finite field, which is impossible. Hence $F$ is algebraic over $\mathbb{F}_{p}$ and it follows that $D$ is algebraic over $\mathbb{F}_{p}$. Now, in virtue of Jacobson's Theorem [12, (13.11), p. 219], $D$ is commutative, a contradiction.

Corollary 2.6. Let $D$ be a division ring of type 2. If $D^{*}$ is finitely generated, then $D$ is a finite field. 
If $M$ is a finitely generated maximal subgroup of $D^{*}$, then clearly $D^{*}$ is finitely generated. So, the next result follows immediately from Corollary 2.6.

Corollary 2.7. Assume that $D$ is a division ring of type 2 . If $D^{*}$ has a finitely generated maximal subgroup, then $D$ is a finite field.

In the same way as in the proof of Theorem 2.5, we obtain the following corollary.

Corollary 2.8. Assume that $D$ is a non-commutative division ring of type 2 with center $F$ and $S$ is a subgroup of $D^{*}$. If $N=S F^{*}$, then $N / N^{\prime}$ is not finitely generated.

Proof. Suppose that $N / N^{\prime}$ is finitely generated. Since $N^{\prime}=S^{\prime}$ and $F^{*} /\left(F^{*} \cap S^{\prime}\right) \simeq$ $S^{\prime} F^{*} / S^{\prime}$, it follows that $F^{*} /\left(F^{*} \cap S^{\prime}\right)$ is a finitely generated abelian group. Now, in the same way as in the proof of Theorem 2.5, we conclude that $D$ is commutative and this is a contradiction.

The following result follows immediately from Corollary 2.8 ,

Corollary 2.9. If $D$ is a non-commutative division ring of type 2 , then $D^{*} / D^{\prime}$ is not finitely generated.

\section{The radicality of subgroups}

In this section we study subgroups of $D^{*}$ which are radical over some subring of $D$. To prove the next theorem we need the following useful property of division rings of type 2 .

Lemma 3.1. Let $D$ be a division ring of type 2 with center $F$ and let $N$ be a subnormal subgroup of $D^{*}$. If for every pair of elements $x, y \in N$, there exists some positive integer $n_{x y}$ such that $x^{n_{x y}} y=y x^{n_{x y}}$, then $N \subseteq F$.

Proof. Since $N$ is subnormal in $D^{*}$, there exists a series of subgroups

$$
N=N_{1} \triangleleft N_{2} \triangleleft \ldots \triangleleft N_{r}=D^{*} .
$$

Suppose that $x, y \in N$ and $K:=F(x, y)$. By putting $M_{i}=K \cap N_{i}, \forall i \in\{1, \ldots, r\}$, we obtain the following series of subgroups:

$$
M_{1} \triangleleft M_{2} \triangleleft \ldots \triangleleft M_{r}=K^{*}
$$

For any $a \in M_{1} \leq N_{1}=N$, suppose that $n_{a x}$ and $n_{a y}$ are positive integers such that

$$
a^{n_{a x}} x=x a^{n_{a x}} \text { and } a^{n_{a y}} y=y a^{n_{a y}} .
$$


Then, for $n:=n_{a x} n_{a y}$ we have

$$
a^{n}=\left(a^{n_{a x}}\right)^{n_{a y}}=\left(x a^{n_{a x}} x^{-1}\right)^{n_{a y}}=x a^{n_{a x} n_{a y}} x^{-1}=x a^{n} x^{-1}
$$

and

$$
a^{n}=\left(a^{n_{a y}}\right)^{n_{a x}}=\left(y a^{n_{a y}} y^{-1}\right)^{n_{a x}}=y a^{n_{a y} n_{a y}} y^{-1}=y a^{n} y^{-1} .
$$

Therefore $a^{n} \in Z(K)$. Hence $M_{1}$ is radical over $Z(K)$. By [6, Theorem 1], $M_{1} \subseteq Z(K)$. In particular, $x$ and $y$ commute with each other. Consequently, $N$ is an abelian group. By $[15,14.4 .4$, p. 440], $N \subseteq F$.

Theorem 3.2. Let $D$ be a division ring of type 2 with center $F, K$ be a proper division subring of $D$, and suppose that $N$ is a normal subgroup of $D^{*}$. If $N$ is radical over $K$, then $N \subseteq F$.

Proof. Suppose that $N$ is not contained in the center $F$. If $N \backslash K=\emptyset$, then $N \subseteq K$. By [15, p. 433], either $K \subseteq F$ or $K=D$. Since $K \neq D$ by the assumption, it follows that $K \subseteq F$. Hence $N \subseteq F$, which contradicts the assumption. Thus, we have $N \backslash K \neq \emptyset$.

Now, to complete the proof of our theorem we shall show that the elements of $N$ satisfy the requirements of Lemma 3.1. To this end, suppose that $a, b \in N$. We examine the following cases:

Case 1: $a \in K$.

Subcase 1.1: $b \notin K$.

We shall prove that there exists some positive integer $n$ such that $a^{n} b=b a^{n}$. Suppose that $a^{n} b \neq b a^{n}, \forall n \in \mathbb{N}$. Then, $a+b \neq 0, a \neq \pm 1$ and $b \neq \pm 1$. So we have

$$
x=(a+b) a(a+b)^{-1}, y=(b+1) a(b+1)^{-1} \in N .
$$

Since $N$ is radical over $K$, we can find positive integers $m_{x}$ and $m_{y}$ such that

$$
x^{m_{x}}=(a+b) a^{m_{x}}(a+b)^{-1}, y^{m_{y}}=(b+1) a^{m_{y}}(b+1)^{-1} \in K .
$$

Putting $m=m_{x} m_{y}$, we have

$$
x^{m}=(a+b) a^{m}(a+b)^{-1}, y^{m}=(b+1) a^{m}(b+1)^{-1} \in K .
$$

Direct calculations give the equalities

$$
x^{m} b-y^{m} b+x^{m} a-y^{m}=x^{m}(a+b)-y^{m}(b+1)=(a+b) a^{m}-(b+1) a^{m}=a^{m}(a-1),
$$

from which we get

$$
\left(x^{m}-y^{m}\right) b=a^{m}(a-1)+y^{m}-x^{m} a .
$$


If $\left(x^{m}-y^{m}\right) \neq 0$, then $b=\left(x^{m}-y^{m}\right)^{-1}\left[a\left(a^{m}-1\right)+y^{m}-x^{m} a\right] \in K$, contrary to the choice of $b$. Therefore $\left(x^{m}-y^{m}\right)=0$ and consequently, $a^{m}(a-1)=y^{m}(a-1)$. Since $a \neq 1, a^{m}=y^{m}=(b+1) a^{m}(b+1)^{-1}$ and it follows that $a^{m} b=b a^{m}$, a contradiction.

Subcase 1.2: $b \in K$.

Consider an element $x \in N \backslash K$. Since $x b \notin K$, by Subcase 1.1, there exist positive integers $r, s$ such that

$$
a^{r} x b=x b a^{r} \text { and } a^{s} x=x a^{s} .
$$

From these equalities it follows that

$$
a^{r s}=(x b)^{-1} a^{r s}(x b)=b^{-1}\left(x^{-1} a^{r s} x\right) b=b^{-1} a^{r s} b,
$$

and consequently, $a^{r s} b=b a^{r s}$.

Case 2: $a \notin K$.

Since $N$ is radical over $K$, there exists some positive integer $m$ such that $a^{m} \in K$. By Case 1 , there exists a positive integer $n$ such that $a^{m n} b=b a^{m n}$.

Theorem 3.2 is closely related to the following conjecture of Herstein in [10]: "For a division ring $D$, given a subnormal subgroup $N$ of $D^{*}$. If $N$ is radical over the center $F$ of $D$, then $N$ is central, i. e. $N \subseteq F$." In Theorem 3.2, the subgroup $N$ is required to be radical over an arbitrary proper division subring $K$ of $D$, which does not necessarily coincide with the center $F$. Notice that $N$ is required to be normal in $D^{*}$. So, the following question seems to be interesting: "For a division ring $D$, given a subnormal subgroup $N$ of $D^{*}$. Is $N$ contained in the center F of $D$, provided it is radical over some proper division subring of $D$ ?"

Finally, we consider the question of the existence of maximal subgroups in $D$ which are radical over $F$. Recall that if $D$ is centrally finite of index different from the characteristic of $F$, then $D^{*}$ contains no such subgroups (see [1, Theorem 5]). Here, we consider the case when $D$ is of type 2 with $[D: F]=\infty$ and we prove that, if $\operatorname{char} F=p>0$, then $D^{*}$ contains no such subgroups.

Theorem 3.3. Let $D$ be a division ring of type 2 with center $F$ such that $[D: F]=\infty$ and char $F=p>0$. Then the group $D^{*}$ contains no maximal subgroups which are radical over F.

Proof. Suppose that $M$ is a maximal subgroup of $D^{*}$ which is radical over $F$. Put $G=$ $D^{\prime} \cap M$. For each $x \in G$, there exists a positive integer $n(x)$ such that $x^{n(x)} \in F$. It follows that $x^{n(x)} \in D^{\prime} \cap F=Z\left(D^{\prime}\right)$. By Theorem 2.3, $Z\left(D^{\prime}\right)$ is torsion, so $x$ is periodic. Thus, $G$ is a torsion group. Since $M^{\prime} \leq G, M^{\prime}$ is also torsion. For any $x, y \in M^{\prime}$, put 
$H=\langle x, y\rangle$ and $D_{1}=F(x, y)$. Then $n:=\left[D_{1}: F\right]<\infty$ and $H$ is a torsion subgroup of $D_{1}^{*} \leq G L_{n}(F)$. By $\left[12,\left(9.9^{\prime}\right)\right.$, p. 154], $H$ is finite. Since $\operatorname{char} F=p>0$, by [12, (13.3), p. 215], $H$ is cyclic. In particular, $x$ and $y$ commute with each other, and consequently, $M^{\prime}$ is abelian. It follows that $M$ is a solvable group. Thus $M$ is a solvable maximal subgroup of $D^{*}$. By [1, Corollary 2, p. 432] and [3, Theorem 6], $[D: F]<\infty$, a contradiction.

\section{References}

[1] Akbari, S.; Mahdavi-Hezavehi, M.; Mahmudi, M.G., Maximal subgroups of $G L_{1}(D)$, J. Algebra 217 (1999), 422-433.

[2] Akbari, S.; Mahdavi-Hezavehi, M., Normal subgroups of $G L_{n}(D)$ are not finitely generated, Proc. Amer. Math. Soc. 128 (2000), 1627-1632.

[3] Akbari, S.; Ebrahimian, R.; Momenaee Kermani, H.; Salehi Golsefidy, A., Maximal subgroups of $G L_{n}(D)$, J. Algebra 259 (2003), 201-225.

[4] P. Draxl, Skew Fields, London Math. Soc. Lecture Note Series 81 (1983), Cambridge Univ. Press.

[5] V. Drensky, Free Algebras and PI-Algebras, Graduate Course in Algebra, SpringerVerlag, Singapore, 2000.

[6] Bui Xuan Hai and Le Khac Huynh, On subgroups of the multiplicative group of a division ring, Vietnam J. Math. 32 (2004), 21-24.

[7] Bui Xuan Hai and Nguyen Van Thin, On subnormal and maximal subgroups in division ring, Southeast Asian Bull. Math. 32 (2008), 931-937.

[8] Bui Xuan Hai and Nguyen Van Thin, On locally nilpotent subgroups of $G L_{1}(D)$, Commun. Algebra 37 (2009), 712-718.

[9] I. N. Herstein, Noncommutative Rings, The Carus Mathematical Monographs.

[10] I. N. Herstein, Multiplicative commutators in division rings, Israel J. Math., 31 (1978), 180-188.

[11] V. K. Kharchenko, Simple, prime and semiprime rings, Handbook of Algebra, Vol. 1, North-Holland, Amsterdam, 1996, 761-812.

[12] T.Y. Lam, A First Course in Noncommutative Rings, GTM 131 (1991), SpringerVerlag. 
[13] Mahdavi-Hezavehi M, Extension of valuations on derived groups of division rings, Commun. Algebra, 23 (1995), 913-926.

[14] L. H. Rowen, Ring Theory, Vol. 1, Academic Press, New York, 1988.

[15] W. R. Scott, Group Theory, Dover Publication, INC, 1988. 